\title{
Ciclo de Vida da Família e Desmatamento na Amazônia: Combinando Informações de Sensoriamento Remoto com Dados Primários*
}

\author{
Marcellus Marques Caldas* \\ Robert Walker* \\ Ricardo Shirota ${ }^{* * *}$ \\ Stephen Perz ${ }^{* * * * *}$ \\ David Skole ${ }^{* * * * * *}$
}

Sumário: 1. Introdução; 2. Trabalhos empíricos sobre desmatamento; 3. A pequena produção na teoria de Chayanov; 4. O modelo empírico proposto; 5. Dados utilizados e definições das variáveis; 6. Estimação do modelo proposto; 7. Resultados e discussão; 8. Conclusões.

Palavras-chave: Amazônia; desmatamento; ciclo de vida; pequena produção; sensoriamento remoto; econometria espacial.

Códigos JEL: C21; D1; O13; Q12.

Este artigo examina as relações entre as características sociodemográficas das famílias de pequenos produtores na Amazônia e a hipótese do ciclo de vida no processo de tomada de decisão de desmatar. A análise foi conduzida combinando informações de sensoriamento remoto e de sistema de informações geográficas com dados primários de 153 pequenos produtores estabelecidos ao longo da rodovia Transamazônica. Análises de regressões foram efetuadas juntamente com testes de autocorrelação espacial. Os resultados levaram à conclusão de que as características sociodemográficas das famílias, bem como fatores institucionais e de mercado, influenciam a tomada de decisão de uso da terra. Esses resultados permitem concluir ainda que, apesar de não serem muito populares entre os cientistas sociais brasileiros, os dados obtidos através das imagens de satélite podem ser muito úteis neste tipo de estudo. Além disso, a pesquisa aqui apresentada indica que dados sociodemográficos da

\footnotetext{
*Artigo recebido em jan. 2001 e aprovado em out. 2002.

${ }^{* *}$ Center for International Development, Harvard University (EUA).

*** Departament of Geography, Michigan State University (EUA).

${ }^{* * * *}$ Departamento de Economia, Administração e Sociologia, Esalq/USP.

${ }^{* * * * *}$ Departamento de Sociologia, University of Florida (EUA).

****** Basic Science and Remote Sensing Initiative, Departement of Geography, Michigan State University (EUA).
} 
família, bem como os de mercado, podem resultar em problemas de má especificação do modelo. O mesmo se aplica a modelos que não incorporem análises espaciais.

This paper examines the relationships between the sociodemographic characteristics of small settlers in the Brazilian Amazon and the life cycle hypothesis in the process of deforestation. The analysis was conducted combining remote sensing and geographic data with primary data of 153 small settlers along the Transamazon Highway. Regression analyses and spatial autocorrelation tests were conducted. The results from the empirical model indicate that sociodemographic characteristics of households, as well as institutional and market factors, affect the land use decision. Although remotely sensed information are not yet very popular among Brazilian social scientists, these results confirm that they can be very useful for this kind of study. Furthermore, the research presented by this paper strongly indicates that family and sociodemographic data, as well as market data, may result in misspecification problems. The same applies to models that do not incorporate spatial analysis.

\section{Introdução}

Dada a diversidade ecológica e riqueza da floresta amazônica, o desmatamento daquela área tem atraído a atenção da comunidade científica nas últimas décadas, principalmente devido às possibilidades de extinção de espécies e de aquecimento global.

O desmatamento tem várias causas, entre elas as políticas governamentais para a região, abrangendo a construção de estradas, a extração madeireira, a mineração, a criação de fazendas agropecuárias e a migração de pequenos produtores (Fearnside, 1985, Mahar, 1989, Browder, 1988, Serrão e Homma, 1993, Serrão et alii, 1996, McCracken et alii, 1999). Apesar dessas pesquisas, pouco se sabe sobre o atual processo de decisão que leva ao desmatamento. Muito embora algumas pesquisas abordem o comportamento maximizador de lucro por parte dos produtores da região, a maioria das análises é realizada em macroescala, com algumas exceções (Jones et alii, 1995, Pichón, 1993, 1997). Conseqüentemente, ainda não se tem uma visão estatisticamente detalhada do desmatamento na região.

Este artigo tem como objetivo principal apresentar a implementação de um modelo estatístico de desmatamento fundamentado numa visão mais complexa do que a dos empregados nas pesquisas anteriores, usando unidades individuais no processo de decisão, em contraste com os dados de unidades geográficas agregadas 
(Reis e Guzman, 1994, Pfaff, 1999).

O foco de atenção encontra-se nos pequenos produtores das áreas de colonização da Amazônia, considerados um dos grandes responsáveis pelos desmatamentos (Walker et alii, 2000). Este artigo vai além das análises empíricas já realizadas sobre desmatamento em áreas de fronteira, principalmente metodologicamente, quando combina informações de dados primários no nível de família com informações de sensoriamento remoto. Contudo, a abordagem já foi utilizada por outros autores, tais como McCracken et alii (1999) e Walker et alii (2000). Entretanto, a abordagem de McCracken foi altamente descritiva e as variáveis socioeconômicas utilizadas na estimação (distância do mercado e tempo de residência na propriedade) não foram teoricamente derivadas. Por outro lado, o trabalho de Walker foi limitado pelo tamanho da amostra $(n=32)$. Neste artigo, utiliza-se uma amostra de 158 observações e uma abordagem centrada na teoria da economia camponesa combinada com a teoria da produção.

O artigo está organizado da seguinte forma: na seção 2, discutimos a evolução da teoria econômica sobre desmatamento em áreas tropicais; na seção 3 analisamos o comportamento do produtor com base na teoria camponesa de Chayanov; na seção 4, apresentamos o modelo proposto, combinando noções de acessibilidade ao mercado com modelos de agricultura familiar de Chayanov; a seção 5 apresenta os dados utilizados com as definições das variáveis; a seção 6 estima o modelo proposto, enquanto a seção 7 apresenta e discute os resultados; a seção 8 conclui o artigo.

\section{Trabalhos Empíricos sobre Desmatamento}

Muitos trabalhos sobre desmatamento foram elaborados desde a contribuição seminal de Allen e Barnes (1985) e seria impossível fazer uma completa revisão aqui. ${ }^{1}$ Contudo, consideramos esse trabalho o ponto de partida, a fim de estabelecer o contexto das análises focando modelos explicitamente espaciais com dados desagregados.

As análises iniciais tentam explicar o desmatamento utilizando escalas agregadas, nas quais o desmatamento estimado ocorre ao longo de um período, $Y$, como função de variáveis socioeconômicas e físicas, $X$, tal que $Y=f(X)$. Nessas pesquisas, os dados eram obtidos para unidades agregadas, tais como estados e municípios, a partir de censos para área total desmatada e de indicadores socioeconômicos (Allen e Barnes, 1985, Rudel, 1989). Pesquisas mais atuais abordam

\footnotetext{
${ }^{1}$ Para mais detalhes, ver Kaimowitz e Angelsen (1998).
} 
a questão utilizando dados de desmatamento obtidos através de análises de imagens de satélite e processados em sistemas de informações geográficas (SIGs), que também geram informações de variáveis independentes (Chomitz e Gray, 1996, Mertens e Lambim, 1997, 2000). Muito embora geógrafos, sociólogos e ecologistas tenham elaborado os primeiros modelos estatísticos de desmatamento, especificações formais baseadas na teoria comportamental só foram desenvolvidas apos a inferência de economistas, que inicialmente conceituaram o processo de desmatamento como uma expressão da demanda derivada por terra, através do convencional problema de maximização da firma e necessidade de fatores de produção (Panayotou e Sungsuwan, 1994, Reis e Guzman, 1994).

Com a disponibilidade de dados altamente desagregados e de medidas de mudança de cobertura vegetal, observadas em nível de pixels, o modelo operacional muda de configuração. Muito embora a maximização de lucro permaneça como o principio direcionador, a unidade de decisão agora aborda parcelas de terras individuais e mudanças discretas entre categorias de cobertura da terra baseadas em um rank de um conjunto finito de alternativas.

Por exemplo, para Bockstael (1996) a probabilidade de desenvolver uma parcela de terra, $t$, é:

$$
\operatorname{Prob}(\text { desenvolver })=\operatorname{prob}\left(V_{t D t p}+\epsilon_{t D t p}>V_{t N d t p}+\epsilon_{t N d t p}\right)
$$

onde $V$ é o lucro líquido decorrente do desenvolvimento $(D)$ da parcela versus o não-desenvolvimento $\left(N_{d}\right)$ no tempo $(t p)$, e $\epsilon$ é uma variável aleatória. Chomitz e Gray (1996) argumentam que um conjunto de usos da terra $T$, alternativos ao não-desenvolvimento, fornecem potenciais rents, $R_{i},(i \epsilon T)$ definidos para preços e produtos. Assim, o uso da terra (ou cobertura) que atualmente ocorre em uma parcela é $t$, quando $R_{t}>R_{i}$, para todo $i \neq t$. Isto leva a uma equação logística quando $R$ é decomposto em componentes aleatórios e fixos, e a variável aleatória é especificada numa forma apropriada. Assim como Bockstael (1996), a abordagem de Chomitz e Gray (1996) é espacialmente explícita, com variáveis independentes, incluindo medidas de distância, geradas por meio de SIGs. ${ }^{2}$

A maximização do lucro (ou rent) constitui a fundamentação comportamental para a maioria dos modelos espacialmente explícitos. Tal hipótese é defensável para explicações de uso da terra sob paradigmas de bid rent (Alonso, 1964), quando direitos de propriedades estão bem definidos e o meio ambiente econômico está livre de choques. Entretanto, o meio ambiente institucional de áreas de fronteira, como no caso da Amazônia, é profundamente diferente da realidade do Primeiro

\footnotetext{
${ }^{2}$ Veja também Nelson e Hellerstein (1997).
} 
Mundo e pode não ser favorável às formas puras de maximização de lucro. Mueller et alii (1994) têm descrito o impacto da insegurança da propriedade da terra na Amazonia e o que isso significa em termos de decisões agrícolas. Níveis reduzidos de investimentos, devido ao curto horizonte de tempo, e a impossibilidade de usar a terra como garantia de empréstimos têm forte efeito sobre os padrões de uso da terra.

Uma possível resposta dos pequenos produtores ao problema da insegurança da terra está relacionada à aversão ao risco, e isso é confirmado pelas observações empíricas para o Brasil (Dillon e Scandizzo, 1978, Homma et alii, 1996, Faminow et alii, 1999), nas quais pelo menos uma abordagem teórica para o caso do desmatamento na Amazônia está sendo desenvolvida, combinando mudanças nos sistemas agrícolas (desde florestas extrativas sustentáveis até sistemas com pecuária) com o aumento na incerteza da renda gerada com florestas, devido, principalmente, ao fogo e a invasões de terra (Homma et alii, 1996).

Walker et alii (2002) adaptam o modelo de Homma et alii (1996) com relação à escolha dos sistema de produção e desenvolvem uma abordagem baseada na aversão ao risco. Esses autores consideram a utilidade associada ao sistema de produção, como pecuária, produção de culturas perenes ou um sistema de produção de culturas anuais. Cada sistema é capaz de gerar uma renda, I, e uma utilidade, $U(I)$. Se $I$ é uma variável aleatória afetada pelas condições locais e pelo tipo de sistema, e se a utilidade é função da estrutura da família, $h$, então a utilidade pode ser escrita como $U(h ; I)$, onde $I$ possui uma função de densidade, $f_{i}(h ; I)$, específica para cada sistema. Contudo, dada a natureza aleatória da renda, cada sistema agrícola possui uma "certeza equivalente", definida em termos práticos como o montante de renda mínimo que um produtor aceitaria para abandonar a atividade de risco em questão. Assim, a "certeza equivalente" pode ser interpretada como o valor que o indivíduo coloca em um sistema agrícola, sujeito a choques aleatórios (por exemplo, aumentos repentinos das taxas de juros), dada a sua atitude de risco. Em termos formais, pode-se defini-la como (DeGroot, 1970, Ellis, 1993):

$$
U\left(C E_{i}\right)=f U(h ; I) f i(h ; I) d I
$$

Walker et alii (2002) usam a certeza equivalente para definir uma função de utilidade aleatória, tal que:

$$
U(i)=C E_{i}+\epsilon_{i}
$$

onde $\epsilon_{I}$ representa uma variável aleatória para os efeitos não observados. Portanto, a probabilidade de que o sistema $I$ seja o selecionado pode ser escrita como: 


$$
P[U(i)>U(t)]
$$

O que é idêntico, em termos operacionais, aos modelos de maximização de lucro de Bockstael (1996), Chomitz e Gray (1996), e Nelson e Hellerstein (1997), para um conjunto finito de sistemas agrícolas alternativos.

\section{A Pequena Produção na Teoria de Chayanov}

Como parte do tratamento de aversão ao risco, o modelo de Walker et alii (2002) necessariamente utiliza definições da teoria da economia camponesa ( $p e-$ asant economy), uma teoria em nível de família explicando as relações entre a estrutura da família e o uso da terra, na presença de mercados não consolidados. Nesse contexto, o modelo da teoria da economia camponesa é de fundamental importância para esta discussão.

A teoria da pequena produção agrícola (Singh et alii, 1986) tem sua base nos estudos de Chayanov sobre a pequena produção familiar na Rússia após a Revolução de Outubro de 1917 (Thorner et alii, 1986).

Para Chayanov, o conceito de família camponesa estava atrelado à dependência ao trabalho familiar a às práticas agrícolas que visavam a subsistência. Além disso, ele assumia que a família camponesa russa tinha acesso flexível à terra e mantinha propriedades de diferentes tamanhos. Chayanov acreditava que a economia camponesa seria mais bem entendida através da organização interna da família. Para ele, sob essas condições de acesso à terra, as características demográficas da família influenciavam a atividade econômica, suas relações de trabalho e o uso da terra. Assim, ele procurava explicar as diferenças em área cultivada em termos da estrutura da família, diferenciando-as pelo estágio no ciclo de vida.

Chayanov desenvolveu uma descrição empírica do ciclo de vida da família, na qual o tamanho da família aumentava e diminuía ao longo do ciclo de vida. Ele interpretava essas mudanças em tamanho como mudanças no número de trabalhadores e de consumidores. A relação entre o número de trabalhadores e o de consumidores foi denominada relação (ou taxa) de dependência. Para Chayanov, essa relação inicia-se com um valor igual a 1 em uma jovem família, composta de um homem e uma mulher, na qual o número de trabalhadores e consumidores está em equilíbrio. Contudo, com o passar dos anos e o nascimento dos filhos, este equilíbrio é afetado em favor do número de consumidores. Nesse estágio, devido à limitação de mão-de-obra disponível para o trabalho agrícola, a quantidade de terra utilizada na agricultura é pouca, e a família concentra-se na produção de produtos 
básicos de subsistência. Mais tarde, com o "amadurecimento" da família, e com as crianças entrando em idade de trabalho, a relação de dependência aumenta, permitindo uma expansão da área cultivada. Em decorrência disso, Chayanov estabeleceu que, durante o ciclo de vida da família, aumentos na relação de dependência levam a uma maior atividade econômica, com maior utilização do trabalho e, inclusive, com a própria exploração do trabalho familiar.

Chayanov procurou formalizar seu pensamento dentro do conceito de salário subjetivo, que pode ser ligado às curvas de indiferença da teoria do consumidor (Ellis, 1993). Cabe ressaltar que a teoria da economia camponesa vem sendo adaptada desde o século passado (Nakajima, 1969, Singh et alii, 1986). Entretanto, apesar de a teoria de Chayanov fornecer as fundamentações teóricas para a análise da relação entre o uso da terra e as características demográficas da família, existem várias hipóteses dentro de sua concepção que dificultam uma direta aplicação ao contexto Amazônico brasileiro (Walker e Homma, 1996). Por exemplo, em seu modelo, Chayanov assume que não existe mercado de trabalho. Assim, as famílias não podem contratar nem vender sua força de trabalho. Assume também que as famílias têm limitadas possibilidades de geração de renda ou investimento fora da propriedade. Além disso, seu modelo considera a existência de um sistema de produção uniforme, variando apenas quanto ao tamanho da propriedade (Thorner et alii, 1986).

Todavia, nenhuma dessas hipóteses se mantém no caso amazônico. O mercado de trabalho existe e as famílias de pequenos produtores regularmente contratam mão-de-obra ou vendem sua força de trabalho, ou até mesmo trocam dias de trabalho. Por outro lado, na Amazônia existem áreas em que famílias vivem próximo do nível de subsistência. Destarte, o capital e o crédito agrícola estão disponíveis, apesar de difícil acesso, com parte da produção freqüentemente vendida no mercado.

Assim, como forma de criar o pano de fundo, é conveniente construir inicialmente um arcabouço teórico baseado nos trabalhos do Centro Agro-Ambiental do Tocantins (1992), de Walker e Homma (1996) e de McCracken et alii (1999). Todos esses trabalhos centram-se na noção de ciclo de vida, através do qual a estrutura da família segue uma série de estágios, em um processo evolucionário que tem implicações para a tomada de decisão de uso da terra. Os estágios são determinados principalmente pela idade do chefe da família, pelo montante de trabalho familiar disponível, pelo número de dependentes (consumidores), pelo uso do trabalho contratado e pela renda gerada fora da propriedade.

Em geral, o cenário começa com a chegada de uma jovem família a uma parcela de terra em áreas de fronteira. Com o passar do tempo, o chefe da família amadu- 
rece e por meio da experiência e experimentação é capaz de melhorar suas práticas agrícolas. Ao mesmo tempo, o número de crianças aumenta, aumentando a demanda por alimento e pelo trabalho familiar. Contudo, à medida que as crianças crescem e passam a fazer parte da força de trabalho familiar, a propriedade pode expandir-se e novos sistemas agrícolas podem ser implementados: conseqüentemente, a aversão ao risco diminui. Por outro lado, à medida que o chefe de família envelhece, as atividades também podem contrair-se, ou continuar a crescer caso os filhos continuem na propriedade construindo o patrimônio da família. Para essas famílias, a vinda para as áreas de fronteira são acompanhadas de uma expectativa de que estas áreas deixarão de ser de fronteira e serão incorporadas às regiões agrícolas já estabelecidas, com mercados de insumos, produtos e capital.

\section{O Modelo Empírico Proposto}

Este artigo apresenta um modelo baseado, em parte, na teoria da economia camponesa, particularmente na relação entre o trabalho familiar e a magnitude da atividade econômica da família. O modelo desenvolve-se a partir da abordagem de sistemas agrícolas estabelecida por Walker et alii (2002). Contudo, diferentemente de Walker et alii (2002), o modelo desenvolvido aqui estima uma equação de demanda derivada por terra, em nível de propriedade, em contraste com as escolhas discretas de sistemas agrícolas desenvolvidas por Walker. Neste caso, o modelo segue as formas estabelecidas por Panayotou e Sungsuwan (1994) e Reis e Guzman (1994). A diferença é que o modelo teórico é aplicado e estimado para unidades de decisão individuais (lotes), e não unidades agregadas, e desenvolvido em termos de variáveis observadas. Portanto, a abordagem pode superar erros de especificações inerentes ao uso de dados agregados (Walker et alii, 2000).

$\mathrm{Na}$ teoria do consumidor assume-se a busca pela otimização de uma função de bem-estar (ou utilidade) oposta ao lucro, muito embora a maximização do lucro seja necessária para a maximização do bem-estar na presença de mercados (Singh et alii, 1986). A função de utilidade utilizada neste artigo é similar à de Walker (1999):

$$
U(l, s, f)
$$

onde $l$ representa o lazer, $s$ a subsistência, e $f$ as características da família. Esta formulação é diferente da de Walker (1999), dado que inclui $f$, para medir o impacto da estrutura da família sobre as preferências. A família maximiza essa função sujeita a um conjunto de restrições. A primeira restrição está relacionada ao trabalho familiar, ou: 


$$
l+w=\theta
$$

onde $w$ representa o trabalho familiar e $\theta$ é o patrimônio (endowment) da família. A subsistência é gerada através de uma função de produção com dois fatores trabalho $(w)$ e terra $(T)$ - ou:

$$
s=f(d, k, w, T)
$$

onde $d$ representa a distância e $k$ é o capital da família, tomados como variáveis exógenas. Desenvolvendo a função de produção como uma função de Leontief (Walker, 1999), temos:

$$
f(d, k, w, T)=g(d, k) \min (a w, b T)
$$

Assumindo uma alocação eficiente de terra e trabalho, podemos simplificar a expressão, de tal forma que:

$$
f(d, k, w, T)=g(d, k) v(w)
$$

onde $T=\theta w$, e $\theta$ é um coeficiente fixo. Pode-se reduzir o problema de maximização de forma a encontrar um valor de $w$ que maximiza:

$$
U[\theta-w ; g(d, k) v(w) ; h]
$$

Assim, a condição de primeira ordem para uma solução interior será:

$$
-U_{1}+U_{s} g(d, k) v^{\prime}(w)=0
$$

e, por meio do teorema da função implícita, pode-se resolver o problema para $w$, de tal modo que:

$$
\begin{gathered}
w=q(\theta, d, k, h) \\
T=\theta q(\theta, d, k, h)
\end{gathered}
$$

Esta última equação é utilizada para estimar os resultados, usando dados primários em nível de família e dados de desmatamento em nível de propriedade, obtidos de sensoriamento remoto. Ressalta-se que a demanda derivada por terra difere das especificações testadas em outros trabalhos, por incluir variáveis que refletem a estrutura da família. Destarte, mantém-se a variável distância, utilizada em outros trabalhos com dados agregados, com o intuito de captar os preços dos 
fatores, fundamentais para equações de demanda de fatores. Contudo, ressalta-se que esta variável é mantida no modelo proposto mesmo não se tendo fornecido argumentações teóricas de como afeta a função de produção. O raciocínio para a manutenção dessa variável consiste em supor que, mesmo no caso da produção de subsistência (ou consumo), haverá uma troca, ainda que pequena, no mercado, e que esta troca é afetada pela distância do mercado. Matematicamente seria possível representar isso como:

$$
f=z(k, w, T)-h(d)
$$

onde $z$ é a produção e $h(d)$ é uma forma de "custos" de transporte. ${ }^{3}$

\section{Dados Utilizados e Definições das Variáveis}

Os dados utilizados na pesquisa apresentada neste artigo foram gerados através de levantamento de campo efetuado no município de Uruará, no estado do Pará, uma fronteira de colonização originariamente aberta por volta dos anos 1970 ao longo da rodovia Transamazônica, e mediante classificação de imagens de satélites. O projeto de colonização de Uruará foi escolhido para a pesquisa por ser composto por uma população de produtores provenientes dos programas de ocupação oficial e, também, por apresentar sinais de ocupação espontânea, com diversificação de culturas anuais e perenes, pastagens e exploração madeireira, e produtores usando tecnologias de derrubada e queima com posterior abandono da terra.

Uruará está localizado entre os municípios de Altamira e Ruropólis, com uma população de aproximadamente 37.395 habitantes (IBGE, 1996). Para o estudo aqui apresentado, foi efetuado um pré-teste, tendo sido entrevistada uma amostra de 261 proprietários em julho de 1996, cobrindo 347 lotes de 100 hectares cada, ao longo da rodovia principal e dos travessões. ${ }^{4}$ Desde os anos 1970, ocorreram algumas concentrações de posse da terra na região, o que explica a diferença entre o número de proprietários e o de lotes.

Muito embora grandes fazendas já apareçam na região, a pesquisa de campo cobriu apenas pequenos produtores. Três equipes, com dois integrantes cada, fizeram o levantamento de campo ao longo de um mês. Os questionários utilizados abordaram questões relacionadas a estrutura da família, situação socioeconômica e sistemas agrícolas.

\footnotetext{
${ }^{3}$ Este tipo de abordagem é similar às utilizadas por Fujita et alii (1999).

${ }^{4}$ Travessões são estradas que cortam a rodovia principal de norte a sul.
} 
As características gerais das famílias amostradas são consistentes com os dados da economia camponesa de Chayanov (Ellis, 1993, Walker et alii, 2002). As famílias amostradas consomem a maior parte do feijão (70\%) e milho(60\%) que produzem e tendem não contratar trabalho assalariado (somente 28 dias por ano de trabalho assalariado). O tamanho médio da família está em torno de 7,12 indivíduos, e há cerca de 2,85 crianças por família. O chefe da família tem em média 47 anos e um tempo médio de escola de 2,02 anos.

A variável dependente, área total desmatada para lotes individuais, foi gerada da seguinte forma: com o software de informações geográficas ArcInfo foram digitalizadas todas as propriedades contidas no mapa original da área de colonização de Uruará fornecido pelo Incra. Esse procedimento objetivou estabelecer a localização precisa de cada propriedade amostrada, uma vez que conheciam-se os endereços de cada propriedade visitada, bem como dados de posição geográfica (GPS).

Dos 261 pequenos produtores amostrados, apenas 163 puderam ser localizados no mapa digitalizado, totalizando 219 lotes. A diferença entre a amostra original de 261 pequenos produtores para a amostra digitalizada de 163 decorreu de três fatores principais: primeiro, alguns produtores estavam assentados em lotes tão distantes da rodovia Transamazônica que nem constavam no mapa original fornecido pelo Incra na época da coleta dos dados e, conseqüentemente, não tiveram suas propriedades localizadas no mapa digitalizado; segundo, às vezes vários produtores eram entrevistados num mesmo local, sem o uso do mapa do Incra, o que dificultou a exata localização da propriedade; finalmente, alguns produtores não sabiam corretamente seus endereços.

O processo de localização das propriedades amostradas no mapa digitalizado envolveu as fases descritas a seguir.

Primeiramente, efetuou-se uma compatibilização das informações fornecidas pelos produtores (número do lote, número da gleba, número do travessão) com as informações contidas no mapa fornecido pelo Incra. Em seguida, com base no mapa do Incra, identificaram-se as propriedades amostradas no mapa digitalizado.

Utilizando-se os mapas digitalizado e do Incra, realizaram-se duas viagens à área em estudo para a coleta de dados de posições geográficas (GPS), com o intuito de conferir a exata localização das propriedades digitalizadas - a primeira ida ao campo ocorreu em julho de 1999 e a segunda em julho de 2000 (figura 1). 
Figura 1

Mapa digitalizado da área de colonização de Uruará ao longo da rodovia Transamazônica

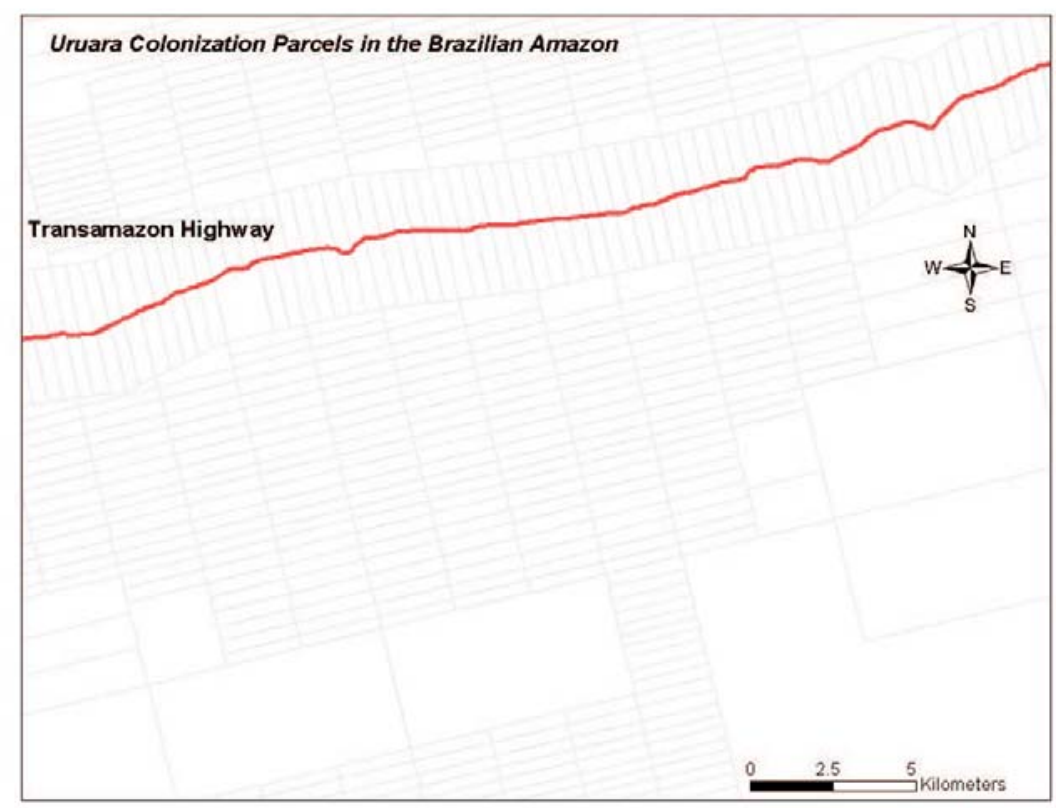

A seguir, foram adquiridas imagens de satélite LandSat5 (através do projeto Nasa LandSat Pathfinder do Basic Science and Remote Sensing Initiative Laboratory da Michigan State University) da região de Uruará (órbitas 226 e 227, ponto 62), para os anos de 1996 e 1997. Todavia, foram utilizadas apenas as imagens do ano de 1997, uma vez que não apresentavam problemas de nuvens sobre a área em estudo, o que não ocorreu em 1996.

Na penúltima etapa, classificaram-se e identificaram-se sete classes temáticas para o ano de 1997 (floresta, desmatamento, cerrado, água, nuvem, sombra de nuvem e floresta secundária). Finalmente, foi efetuada uma sobreposição do mapa digitalizado da área de colonização de Uruará sobre a imagem de 1997. Essa sobreposição espacial possibilitou o cálculo da magnitude do desmatamento para cada lote, definida como a soma das áreas classificadas como desmatamento ou floresta secundária (figura 2). 
Figura 2

Mapa digitalizado da área de colonização de Urará sobreposto em uma imagem de Landsat

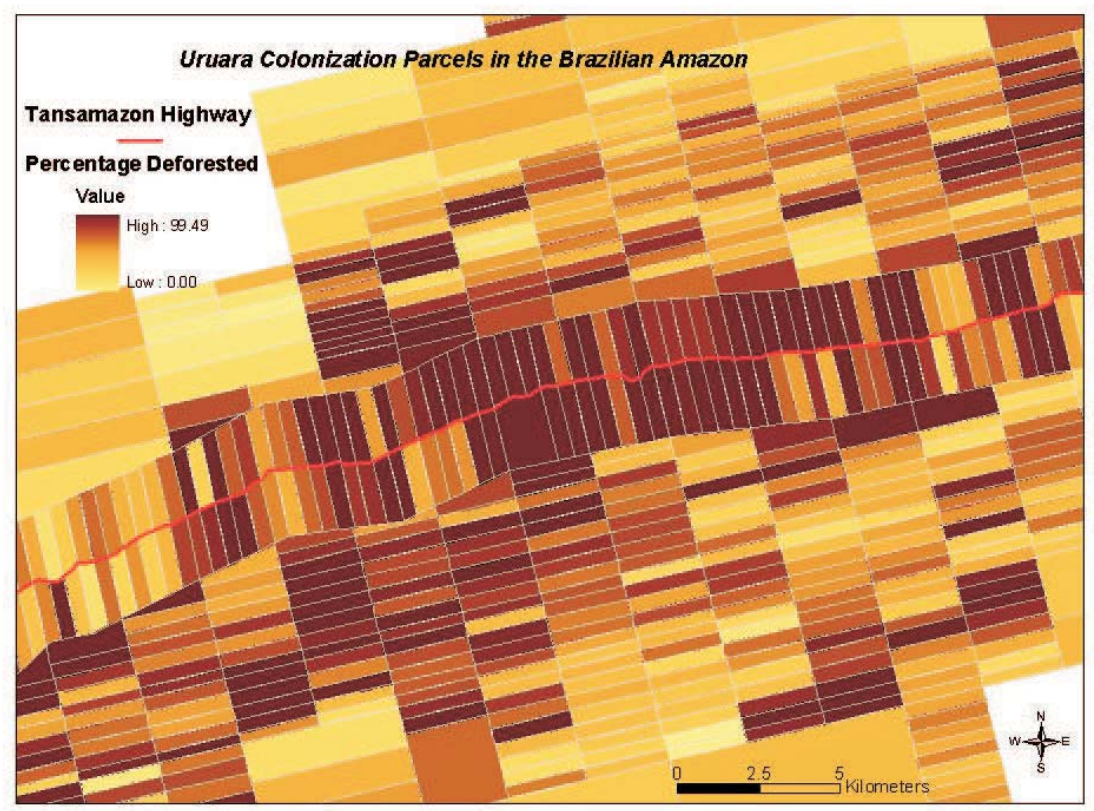

As variáveis independentes, obtidas através do levantamento de campo, são as indicadas nas equações (12) e (13) do modelo proposto. Essas variáveis representam a força de trabalho familiar, o capital inicial da família, a distância da principal rodovia de acesso ao mercado e medidas de dependência. Outras variáveis incluem a idade do chefe da família (representando o capital humano) e o tempo de residência na propriedade, bem como características do meio ambiente institucional (uso de crédito e tipo de posse da propriedade).

\section{A Estimação do Modelo Proposto}

O modelo estatístico considerado neste artigo possui a seguinte forma funcional:

$$
\Upsilon_{i}=X_{i} \beta+\epsilon_{i}
$$

onde $\Upsilon_{i}$ representa o desmatamento que ocorre no lote $i, X_{i}$ é um vetor de variáveis explicativas, $\beta$ é um vetor de coeficientes e $\epsilon_{i}$ é um vetor de termos aleatórios. 
A principal contribuição dos modelos de regressão está centrada na identificação das variáveis fortemente associadas ao desmatamento, assumidas como as principais forças que influenciam o desmatamento. Contudo, deve-se ter cuidado ao extrapolar os resultados no espaço ou no tempo, pois é muito possível que um modelo que se ajuste bem a determinada região não reproduza os mesmos resultados em outra.

Da mesma forma, enquanto os modelos de regressão podem tratar adequadamente a dimensão tempo do processo que representam, são intrinsecamente não-espaciais, ou seja, não consideram a distribuição espacial dos elementos em uma área e os efeitos adjacentes. Isto quer dizer que as análises cross-section em níveis regional ou nacional são baseadas na hipótese não-realista de que o desmatamento em dada unidade administrativa é preliminarmente função de fatores daquela região. Conseqüentemente, quando, em modelos de regressão, se utilizam dados advindos de coordenadas geográficas, deve-se considerar a possibilidade da influência de efeitos espaciais. Assim, assumimos que a autocorrelação espacial pode ser um problema, uma vez que adoções de tecnologia em um determinado lote podem influenciar adoções em outros lotes (contagion effect) (Casetti e Semple, 1969), bem como a não-inclusão de variáveis (como qualidade do solo) que provavelmente apresentam correlação positiva no espaço.

A autocorrelação espacial ou, mais genericamente, a dependência espacial, pode ser definida como a situação em que a variável dependente ou o termo de erro em cada localidade estão correlacionados com as observações da variável dependente ou os valores do termo de erro em outras localidades.

Em termos gerais, pode-se formalmente definir a autocorrelação espacial como:

$$
E\left(y_{i} y_{j}\right) \neq 0
$$

ou

$$
E\left(\epsilon_{i} \epsilon_{j}\right) \neq 0
$$

para vizinhanças $i$ e $j$. Contudo, este tipo de especificação é muito genérico para permitir a estimação de $N$ vezes $(N 1)$ interações das $N$ observações. Dessa forma, a estrutura da dependência espacial é representada por uma matriz de ponderação espacial $(W)$, que reduz o número de parâmetros desconhecidos a 1 , isto é, ao coeficiente da associação espacial num processo espacial auto-regressivo.

A dependência espacial ou autocorrelação espacial pode ocorrer de duas formas. Na primeira, afeta somente os termos de erro e é, na maioria das vezes, considerada uma perturbação que precisa ser eliminada (Anselin, 1988). Como no caso geral 
de erros não-correlacionados, a autocorrelação espacial dos resíduos não produz estimativas viesadas de mínimos quadrados ordinários, mas altera sua eficiência. Conseqüentemente, a interpretação do nível de significância, que é baseada em testes $t$ e $R^{2}$, é viesada (Anselin e Rey, 1991).

$\mathrm{Na}$ segunda forma, é dada à dependência espacial uma interpretação substantiva, em que a variável de interesse em certa localização é determinada por seus valores em outras. Por exemplo, a área desmatada para o plantio de cacau em uma determinada propriedade pode ter recebido influência de áreas desmatadas em localidades vizinhas, que também plantaram cacau. Assim, um modelo corretamente especificado deveria incluir um termo espacial auto-regressivo, isto é, uma soma ponderada dos valores da variável dependente em outras localizações, ou seja, uma variável dependente espacialmente defasada (Anselin e Rey, 1991).

Dessa forma, uma importante questão em análise empírica está centrada na detecção desses efeitos espaciais e, além disso, em como diferenciar a autocorrelação espacial decorrente de uma perturbação espacial daquela resultante de uma interpretação substantiva (Doreian, 1980, Anselin, 1988).

Assim, um modelo auto-regressivo para termos de erros pode ser formalmente especificado como:

$$
\begin{gathered}
\mathbf{Y}=\beta \mathbf{X}+\epsilon \\
\boldsymbol{\epsilon}=\lambda \mathbf{W} \boldsymbol{\epsilon}+\boldsymbol{\mu}
\end{gathered}
$$

onde: $\mathbf{Y}$ é um vetor $N \times 1$ de observações da variável dependente; $\mathbf{X}$, uma matriz $N \times K$ de variáveis independentes ou exploratórias; $\beta$, um vetor de coeficientes $K \times 1 ; \boldsymbol{\epsilon}$, um vetor de erros $N \times 1$, assumindo serem normalmente distribuídos, mas autocorrelacionados; $\lambda$, o coeficiente espacial auto-regressivo (que mede a intensidade do fenômeno da autocorrelação espacial); $\mathbf{W}$, uma matriz de influência espacial $N \times N ; \boldsymbol{\mu}$, o termo de erro, assumido como normalmente distribuído - $\boldsymbol{\mu}$ $\sim N\left(0, \sigma^{2} I\right)$.

Neste caso, pode-se definir a hipótese nula da seguinte forma:

$$
H_{0}: \lambda=0
$$

ou seja, o termo de erro é não-correlacionado.

A matriz de influência espacial $\mathbf{W}$ expressa, por sua vez, o poder da interação entre cada observação e as observações adjacentes, ou seja, a estrutura dos dados em termos de espaço. Essa matriz pode ser baseada em um coeficiente de contigüidade binária simples $\left(w_{i j}=1\right.$, se $i$ e $j$ tiverem fronteira comum, e $w_{i j}=0$ 
em caso contrário). Convencionou-se que os elementos da diagonal principal dessa matriz são zero, isto é, nenhuma observação é vizinha dela mesma. ${ }^{5}$

Os modelos de autocorrelação espacial freqüentemente são resultado da nãoespecificação de variáveis geográficas importantes, tais como qualidade do solo e tipo de vegetação, que operam mais em grande escala do que em nível micro (por exemplo, lotes). Dessa forma, a correlação espacial nos resíduos de uma regressão pode refletir a omissão de variáveis, a má especificação do modelo ou erros de medida dos dados utilizados.

A segunda forma de autocorrelação espacial é conhecida como modelo autoregressivo de defasagem espacial misto, em que uma defasagem espacial, WY, para a variável dependente é introduzida no modelo de regressão como variável explanatória adicional. Nesse caso, o modelo é similar a um termo auto-regressivo em análises de séries temporais, ${ }^{6}$ muito embora haja várias importantes diferenças que requerem metodologia especializada para estimativa e teste. Formalmente, esse modelo é expresso como:

$$
\mathbf{Y}=\rho \mathbf{W Y}+\boldsymbol{\beta} \mathbf{X}+\boldsymbol{\mu}
$$

onde $\rho$ é o coeficiente espacial auto-regressivo e $\boldsymbol{\mu}$ um vetor $N \times 1$ com erros normalmente distribuídos, com as outras notações permanecendo como antes.

Neste caso, a hipótese nula da ausência de autocorrelação corresponde a:

$$
H_{0}: \rho=0
$$

A impropriedade dos estimadores de mínimos quadrados para modelos que incorporam dependência espacial tem focalizado a atenção na abordagem da máxima verossimilhança como alternativa (Ord, 1975, Anselin, 1988). Neste caso, pode-se adaptar um procedimento interativo desenvolvido primeiramente por Cochrane e Orcutt (1949) em modelos de séries temporais, para o caso de correlações espaciais. Dessa forma, para cada modelo, os coeficientes auto-regressivos são obtidos com estimativa por máxima verossimilhança. O procedimento adaptado de Cochrane e Orcutt consiste em três passos: primeiro, os estimadores de mínimos quadrados ordinários são calculados; os resíduos são, então, utilizados para obter uma estimativa para os coeficientes $\lambda$ e $\rho$ de autocorrelação espacial; finalmente, esses

\footnotetext{
${ }^{5}$ Em análises de regressão, a matriz $\mathbf{W}$ é tipicamente normalizada, isto é, cada elemento da matriz de contigüidade original é dividido pela soma da linha a que pertence, de modo que, na matriz normalizada, a soma de cada linha é igual a 1.

${ }^{6}$ Apesar de a autocorrelação espacial ser, de certa forma, análoga ao problema de correlação serial na análise de séries temporais, a maior complexidade está na direção da influência, já que, na autocorrelação espacial, as variáveis podem influenciar em todas as direções.
} 
coeficientes são substituídos no procedimento de estimativa de máxima verossimilhança.

Uma importante questão na análise espacial empírica está centrada na identificação da presença desses efeitos espaciais, ou seja, como distinguir entre a dependência espacial decorrente de um distúrbio e aquela decorrente da influência de uma interpretação substantiva.

Um teste bem conhecido para autocorrelação espacial em termos de erros da regressão, desenvolvido por Cliff e Ord (1972), baseia-se na generalização da estatística de Moran I para os resíduos da regressão. Neste caso, a estatística de Moran I para a autocorrelação espacial pode ser aplicada utilizando os resíduos da regressão de forma direta. Formalmente, tem-se:

$$
\mathbf{I}=(N / S) \mathbf{e}^{\prime} \mathbf{W e} / \mathbf{e}^{\prime} \mathbf{e}
$$

onde e é o vetor de resíduos dos mínimos quadrados ordinários; $\mathbf{W}$, uma matriz de influência espacial; $N$, o número de observações; $S$, um fator de estandardização igual à soma de todos os elementos da matriz $\mathbf{W}$.

A interpretação desse teste não é tão direta quanto sua aplicação, muito embora seja o mais utilizado na literatura (Anselin e Rey, 1991). Neste caso, a hipótese nula avalia a presença da dependência espacial, e uma precisa expressão para a hipótese alternativa não existe (Anselin e Rey, 1991). A inferência é baseada no valor de $z$ estandardizado, que segue uma distribuição normal (assintoticamente). Intuitivamente, a matriz $\mathbf{W}$ representa o padrão espacial que causa a dependência, mas a natureza do processo estocástico não é especificada. Usualmente, assume-se ser da forma espacial auto-regressiva. O software SpaceStat reporta o valor da estatística de Moran I, o correspondente valor de $z$, e a probabilidade associada de acordo com a destituição normal padrão. Entretanto, segundo Anselin e Rey (1991), o teste de Moran I apenas indica a presença da autocorrelação ou não. Para esses autores, faz-se necessária a distinção entre os dois tipos de autocorrelação espacial, que podem ser detectados pelo teste dos multiplicadores de Lagrange.

Os testes dos multiplicadores de Lagrange para dependência espacial situam-se no domínio da máxima verossimilhança. Têm base nos teoremas de limite central para séries dependentes, que podem ser aplicados ao processo espacial. Esses testes são assintoticamente distribuídos a $\chi^{2}$ e com 1 grau de liberdade (Anselin e Rey, 1991). Assim, o teste de autocorrelação espacial do termo de erro pode ser calculado aplicando-se a seguinte fórmula:

$$
M L_{e r r}=\left[N \mathbf{e}^{\prime} \mathbf{W e} / \mathbf{e}^{\prime} \mathbf{e}\right]^{2}\left[\operatorname{tr}\left(\mathbf{W}^{\prime} \mathbf{W}+\mathbf{W}^{\mathbf{2}}\right)\right]^{-\mathbf{1}}
$$


onde $t r$ representa o traço da matriz, com os outros símbolos permanecendo como antes.

O teste dos multiplicadores de Lagrange para a variável dependente espacialmente defasada pode ser aplicado utilizando a seguinte fórmula:

$$
M L_{\text {lag }}=\left[N \mathbf{e}^{\prime} \mathbf{W} \mathbf{Y} / \mathbf{e}^{\prime} \mathbf{e}\right]^{2}\left[\mathbf{N}(\mathbf{W X b})^{\prime} \mathbf{M}(\mathbf{W X b}) / \mathbf{e}^{\prime} \mathbf{e}+\operatorname{tr}\left(\mathbf{W}^{\prime} \mathbf{W}+\mathbf{W}^{\mathbf{2}}\right)\right]^{-\mathbf{1}}
$$

onde $\mathbf{b}$ representa a estimativa de mínimos quadrados ordinários para $\mathbf{B}$, e $\mathbf{M}$ é uma matriz idempotente. Para Anselin e Rey (1991), não há uma exata distribuição das estatísticas dos multiplicadores de Lagrange, e um atrativo de ambos os testes tem pôr base a utilização das estimativas obtidas pelo cálculo dos mínimos quadrados ordinários.

Duas formas de (15) são consideradas com o intuito de refletir caracterizações operacionais diferentes para a estrutura da família. O modelo 1 usa a medida de dependência elaborada por Chayanov, enquanto o modelo 2 substitui a dependência por um conjunto de variáveis descrevendo a estrutura demográfica da família. A estimação segue a abordagem elaborada por Walker (1999) e Walker et alii (2000), na qual primeiro calculam-se os estimadores de mínimos quadrados ordinários, a seguir utilizam-se os resíduos da regressão para fazer os testes de autocorrelação espacial e, finalmente, uma vez aceita a hipótese de que existe autocorrelação espacial, utiliza-se o procedimento de máxima verossimilhança para a estimativa do modelo apropriado.

\section{Resultados e Discussão}

Neste artigo os diagnósticos para os problemas de autocorrelação espacial foram calculados para dois modelos separadamente; um considerando o tamanho da família e a dependência (modelo 1) e o outro considerando o desmembramento da família em total de homens, mulheres, crianças e idosos (modelo 2). Nos dois casos, os resultados são baseados na amostra contendo informações georreferenciadas (n $=153$ propriedades). A diferença entre a amostra de 163 propriedades digitalizadas e a de 153 utilizada na análise de regressão ocorreu devido às limitações do software de análise espacial SpaceStat, que exige que dados não declarados sejam eliminados da amostra.

Como relatado, os diagnósticos baseados no teste de Moran I apenas indicam se o modelo testado apresenta, ou não, problemas de autocorrelação espacial. Todavia, esse teste não especifica o tipo de problema. Autores como Walker et alii 
(2000), ao estudarem o processo de desmatamento associado a pequenos produtores e pecuaristas na Amazônia, constataram a inexistência de problemas de autocorrelação espacial para vizinhanças de 7 mil, 20 mil e 40 mil metros. Além disso, esses autores ressaltam que não há um meio de se definir a priori as distâncias a serem testadas quando se estabelece a vizinhança.

Assim, a tabela 1 apresenta os testes de Moran I e os dos multiplicadores de Lagrange. Esses testes foram computados para três matrizes de distância, necessárias na implementação da matriz de contigüidade, definidas para as vizinhanças de 10 mil, 20 mil, e 30 mil metros. Os resultados de Moran I revelam que os dois modelos testados apresentam problemas de autocorrelação espacial nas vizinhanças de até 10 mil metros.

Tabela 1

Avaliação dos efeitos espaciais

\begin{tabular}{lcccccccc}
\hline \multicolumn{1}{c}{ Teste } & \multicolumn{3}{c}{ Modelo 1} & & \multicolumn{3}{c}{ Modelo 2} \\
\cline { 2 - 3 } \cline { 6 - 8 } & $10.000 \mathrm{~m}$ & $20.000 \mathrm{~m}$ & $30.000 \mathrm{~m}$ & & $10.000 \mathrm{~m}$ & $20.000 \mathrm{~m}$ & $30.000 \mathrm{~m}$ \\
\hline Teste de Moran I & 2,51 & 0,79 & 0,18 & & 2,45 & 0,36 & $-0,35$ \\
& $(0,012)$ & $(0,430)$ & $(0,856)$ & & $(0,014)$ & & $(0,721)$ & $(0,727)$ \\
Multiplicador de & 3,15 & 0,00 & 0,20 & & 2,95 & 0,07 & 0,67 \\
Lagrange para erros & $(0,076)$ & $(0,943)$ & $(0,650)$ & & $(0,086)$ & & $(0,784)$ & $(0,413)$ \\
Multiplicador de & 5,69 & 0,15 & 0,19 & & 5,59 & 0,05 & 0,28 \\
Lagrange para $l a g$ & $(0,017)$ & $(0,694)$ & $(0,650)$ & & $(0,018)$ & $(0,815)$ & $(0,599)$ \\
\hline
\end{tabular}

Com o intuito de definir o tipo de autocorrelação espacial presente nos resultados, utilizamos os testes dos multiplicadores de Lagrange. Para lotes na vizinhança de até 10 mil metros, os dois modelos apresentam problemas de autocorrelação espacial de erro, bem como de autocorrelação espacial do tipo lag. Contudo, segundo Anselin (1988), em casos como este deve-se optar pelo modelo com maior nível de significância. Assim, os dois modelos apresentam padrões de autocorrelação espacial do tipo lag. Em outras palavras, é provável que o desmatamento que ocorre em determinado lote influencie o desmatamento em outro lote na vizinhança de até 10 mil metros.

Dado que foi possível identificar o tipo de problema espacial existente, a próxima etapa foi a estimação dos dois modelos propostos, através da estimativa de máxima verossimilhança para modelos espacialmente defasados (tabela 2). 


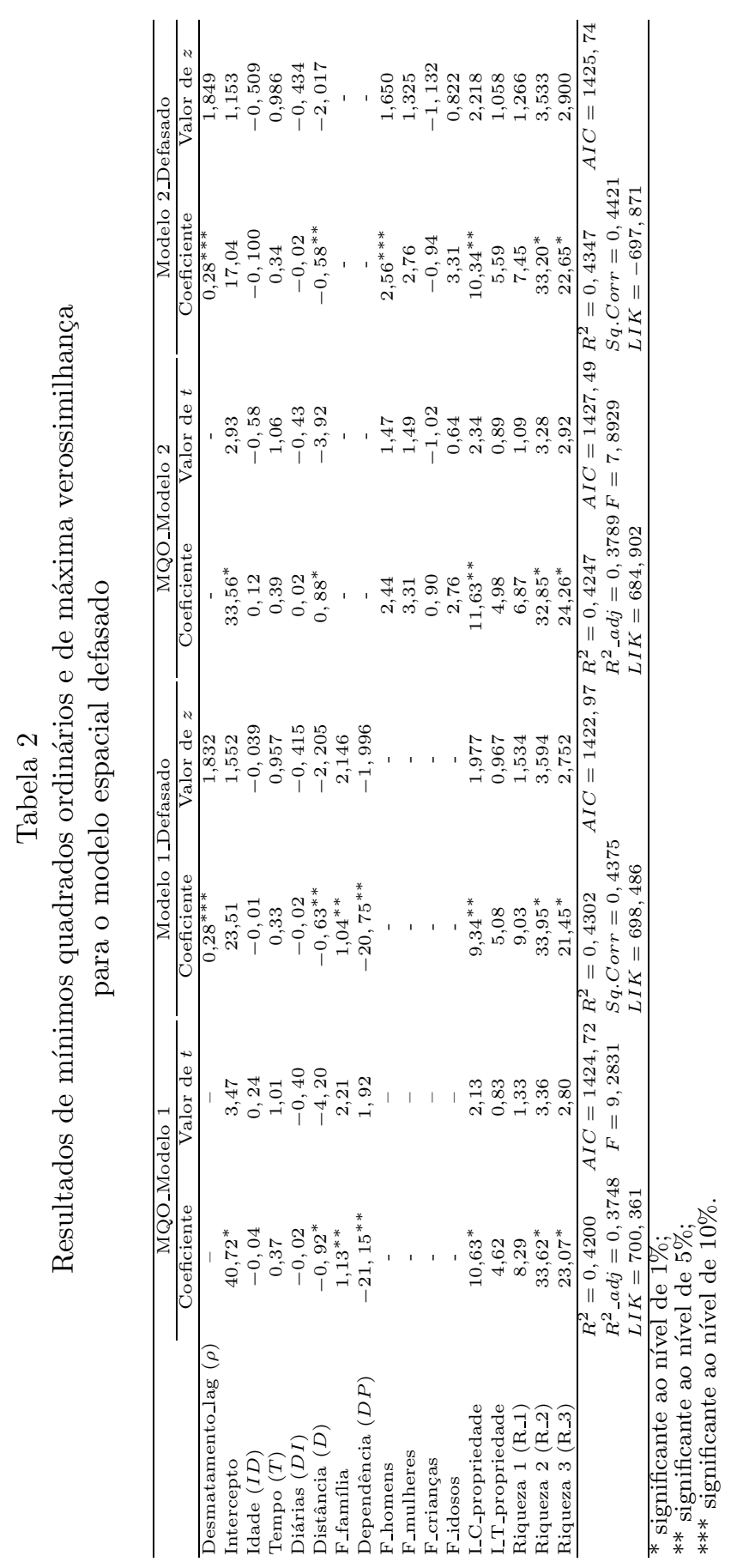


A regressão espacial, para cada modelo de desmatamento propostos, resultou em um $R^{2}$ de $43,02 \%$ e $43,47 \%$, respectivamente. Os resultados são em geral comparáveis. Assim, a essência da discussão se dará mais sobre os resultados gerais e as diferenças entre os modelos 1 e 2, do que sobre o poder dos procedimentos econométricos. Contudo, não se pode esquecer que as estimativas calculadas são não-viesadas.

Para o modelo 1, a variável desmatamento defasado foi significante ao nível de $10 \%$. As variáveis distância, tamanho da família, dependência e crédito na propriedade foram significante ao nível de $5 \%$, e as variáveis riqueza 2 e riqueza 3 foram estatisticamente significantes aos níveis de $1 \%$. Com relação ao modelo 2 , observa-se que não houve variações nos níveis de significância das variáveis analisadas. A variável desmatamento defasado também foi significante ao nível de $10 \%$, juntamente com a variável total de homens na família, as variáveis distância e crédito foram significantes ao nível de $5 \%$, e as variáveis riquezas 2 e 3 , foram significantes aos níveis de 1\%. Portanto, a significância estatística para o coeficiente da variável desmatamento defasado nos dois modelos indica que há presença de agrupamentos de desmatamento.

Nos dois modelos testados, o efeito substantivo da variável acessibilidade ao mercado (representada pela proxy distância) é bastante significativo na tomada de decisão de desmatar. Neste caso, pode-se argumentar que quanto maior a distância em relação à "faixa", 7 maior é a probabilidade de que a terra permanecerá em floresta. Em outras palavras, um quilômetro a mais em direção ao fundo do travessão representa mais de meio hectare a menos em desmatamento. Outros estudos têm considerado o papel que as rodovias e a proximidade do mercado assumem para explicar o desmatamento. Wear e Bolstad (1998), por exemplo, argumentam que o uso da terra e o desmatamento são determinados conjuntamente e que a acessibilidade ao mercado afeta o uso da terra, que por sua vez, determina o desmatamento. Nossa abordagem segue esta linha geral de pensamento, uma vez que as rodovias existem antes das decisões de imigrar.

Dessa forma, é possível constatar que colonos assentados em propriedades mais próximas da "faixa" e, por conseguinte, do mercado, são os que têm maiores áreas desmatadas, com maiores pastagens e culturas perenes. Por outro lado, colonos com pouca acessibilidade ao mercado são mais dependentes de culturas de subsistência.

De uma maneira geral, os resultados apresentados aqui estão em consonância com os de Walker et alii (2002). Segundo estes autores, os colonos localizados mais perto do mercado, na região da rodovia Transamazônica, podem ser caracterizados

\footnotetext{
7 "Faixa" é a denominação atribuída pelos colonos à rodovia Transamazônica.
} 
como "especializados em pecuária", "especializados em perenes com pecuária" ou "especializados em perenes com anuais". Deste ponto de vista, pode-se supor que quanto maior a distância, maiores serão os custos de transporte, menores os preços do produto na propriedade e maior o preço dos insumos, de forma que menores áreas serão desmatadas. Muitos estudos mostram que o desmatamento decresce rapidamente a partir de dois a três quilômetros da rodovia principal, muito embora Liu et alii (1993) e Mamingi et alii (1996) reportarem significantes desmatamentos associados com maiores distâncias em Camarões, nas Filipinas e no Zaire.

As variáveis demográficas também têm papel notável em explicar o desmatamento. Neste caso, verifica-se que famílias com poucos membros são as com maiores probabilidades de estar associadas a maiores áreas com florestas, enquanto uma maior disponibilidade de mão-de-obra estará associada a maiores áreas desmatadas. Neste caso, é comum observar na região que as famílias maiores estão associadas com maiores áreas em perenes, maiores áreas com pastagens e maiores áreas com culturas anuais.

Por outro lado, se desmembrássemos a variável tamanho da família, observaríamos que apenas a variável total de homens na família teria forte significância sobre a área total desmatada. Neste caso, constata-se que os efeitos da força masculina se fazem presentes, pelo menos pelo lado da oferta de mão-de-obra, uma vez que os homens estão mais envolvidos no processo de limpeza da área (desmatamento). Contudo, apesar do efeito de um homem a mais sobre a área total desmatada ser maior, seu nível de significância é menor do que o da variável tamanho da família. Todavia, estes resultados não chegam a surpreender, uma vez que confirmam a natureza intensiva do fator trabalho nas atividades agrícolas empregadas na região, principalmente nas culturas do cacau, café e pimenta-do-reino.

Considerando o efeito da variável dependência sobre a área total desmatada, verifica-se, tal como esperado, que quanto maior a dependência, menor a área desmatada. Portanto, pode-se sugerir que, em termos do modelo proposto, tanto o lado da oferta de mão-de-obra quanto o da demanda (consumo) são significantes na análise da dinâmica de uso da terra. Dessa forma, considerando as variáveis que diretamente refletem estágios do ciclo de vida da família, tais como idade do chefe da família, tempo de residência na propriedade, dependência e tamanho da força de trabalho, apenas as duas últimas parecem influenciar a área desmatada nos dois modelos propostos. Contudo, apesar de a variável tempo de residência não ter sido significante, o resultado não chega surpreender. Godoy et alii (1997) verificam que em Honduras a área total desmatada diminui para famílias indígenas ao longo do tempo, o que pode estar refletindo estágios avançados no ciclo de vida.

Diferentemente de outros estudos, neste a variável diárias não foi significante 
em nenhum dos modelos propostos. É possível que esta variável seja endógena ao modelo, e não exógena, como pressuposto, uma vez que a contratação de mãode-obra assalariada está altamente relacionada ao nível de riqueza da família. Se assim for, quanto maior o nível de riqueza, maior seria a contratação de mão-deobra e, conseqüentemente, maior o desmatamento.

Dado que, nas fases iniciais de consolidação da família na propriedade, algum desmatamento se faz necessário, e tendo em vista que deve ter havido algum dispêndio procuramos, então, analisar os efeitos do nível de riqueza inicial da família na área total desmatada. Tal como esperado, constatamos que nos dois modelos propostos o nível de riqueza inicial é o mais importante fator de influência sobre a área desmatada. Todavia, os resultados sugerem haver um nível mínimo de riqueza a partir do qual sua influência sobre a área desmatada é maior. Por outro lado, os resultados também sugerem que a partir de um determinado nível de riqueza, o efeito sobre o desmatamento é menor.

Muito embora pareça que características sociodemográficas, tais como tamanho da família, total de homens e dependência, sejam importantes para o processo de desmatamento, constata-se que fatores institucionais também são significantes. De fato, a variável crédito é estatisticamente significante nos dois modelos apresentados. Estudos anteriores demonstraram que a disponibilidade de crédito promove o desmatamento, uma vez que permite aos produtores aumentar a área plantada e as pastagens (Monela, 1995, Almeida e Campari, 1995).

\section{Conclusões}

O objetivo principal deste artigo centrou-se no entendimento das forças determinantes do desmatamento que acontece em áreas de colonização ao longo da rodovia Transamazônica. Como objetivo secundário buscamos avançar na metodologia, integrando informações primárias com dados de informações de satélites, mediante a utilização do software SpaceStat, capaz de corrigir problemas de autocorrelação espacial. No que diz respeito ao objetivo secundário, o estudo apresentado no artigo desenvolveu com sucesso análises de regressão do comportamento da família usando a combinação de informações proposta. Alem disso, o estudo ressaltou a importância de considerar problemas de autocorrelação espacial e apontou formas de corrigi-los, no sentido de obter resultados não-viesados. Some-se a isso o fato de que imagens de satélite para levantamento de medidas de desmatamento são mais realistas do que dados extraídos de levantamentos reportados por produtores (Walker et alii, 2000).

Assim como em outros estudos, as análises estatísticas deste apontam para a 
importância do acesso ao mercado com um fator influenciador do desmatamento. Em particular, ressalta que diferentemente dos centros urbanos, na região da rodovia Transamazônica, a partir de certa distância da cidade, os pequenos produtores têm que carregar sua produção até ao mercado e, uma vez que poucos possuem meios de transporte, isso se transforma numa grave restrição ao desenvolvimento dos lotes.

Os resultados demonstram o forte efeito do desmatamento associado com a estrutura da família, em particular com a variável dependência. Esse resultado sugere que os efeitos observados são explicados pela mudança para pastagens, nas quais a redução da aversão ao risco tem papel importante, no momento em que há mudança de sistemas agrícolas de subsistência para sistemas mais orientados para o mercado (Walker et alii, 2002). Alem disso, a demanda de consumo da família exerce notável efeito, indicando que este tipo de abordagem, isto é, modelos que consideram a agricultura familiar, é importante em análises de processos de desmatamento em áreas de colonização. Neste contexto, os resultados sugerem que modelos que omitem dados no nível de família serão afetados por viés de especificação.

Particular atenção deve ser dada à variável crédito, em termos do debate de políticas que promovam o desenvolvimento sustentável. O crédito agrícola é freqüentemente ressaltado como um importante componente em programas de desenvolvimento agrícola. Infelizmente, o efeito da variável crédito sobre o desmatamento é bastante forte. Entretanto, não propomos a não-liberação de crédito como uma abordagem para a conservação ambiental, na medida em que a sustentabilidade social é essencial para a sustentabilidade ambiental de longo prazo. Contudo, este é um problema que deve ser abordado, já que investimentos na agricultura evidentemente aumentarão a demanda por propriedade de terra.

Quando tomados como um todo, os resultados sugerem que fatores demográficos combinados com o meio ambiente econômico afetam as decisões de uso da terra dos colonos. Se assim for, é bem possível que boa parte do desmatamento que ocorre na Amazônia não sofra influência das políticas elaboradas, uma vez que isso pressupõe que o comportamento econômico é orientado somente por considerações econômicas. Destarte, com os novos programas de abertura de estradas e criação de infra-estrutura na Amazônia (Programa Avança Brasil), torna-se cada vez mais importante o entendimento da dinâmica de uso da terra associada aos pequenos agricultores. 


\section{Referências}

Allen, J. C. \& Barnes, D. F. (1985). The causes of deforestation in developing countries. Annals of the Association of American Geographers, 75:163-184.

Almeida, A. L. O. \& Campari, J. S. (1995). Sustainable Settlement in the Brazilian Amazon. Oxford University Press, New York.

Alonso, W. (1964). Location and Land Use. Harvard University Press, Cambridge.

Anselin, L. (1988). Spatial econometrics: Methods and models. The Netherlands, Kluwer, Academics Publishers.

Anselin, L. \& Griffith, D. A. (1988). Do spatial effects really matter in regression analysis? Regional Science Association Papers, (65):11-34.

Anselin, L. \& Rey, S. (1991). Properties of tests for spatial dependence in linear regression models. Geographical Analysis, 23(2):112-31.

Bockstael, N. E. (1996). Modeling economics and ecology: The importance of spatial perspective. American Journal of Agricultural Economics, 78:1168-80.

Browder, J. (1988). Public policy and deforestation in the Brazilian Amazon. In Repetto, R. \& Gillis, M., editor, Public Policies and the Misuse of Forest Resources, page 432. Cambridge University, Cambridge.

Casetti, E. \& Semple, R. K. (1969). Concerning the testing of spatial diffusion process. Geographical Analysis, 1:254-259.

Centro Agro-Ambiental do Tocantins (CAT) (1992). Elementos de análise do funcionamento dos estabelecimentos familiares da região de Marabá. ms.

Chomitz, K. M. \& Gray, D. A. (1996). Roads, land use and deforestation: A spatial model applied to Belize. The World Bank Economic Review, 10:487-512.

Cliff, A. \& Ord, K. (1972). Testing for spatial autocorrelation among regression residuals. Geographical Analysis, 14:267-84.

Cochrane, D. \& Orcutt, G. H. (1949). Applications of least square regressions to relationships containing autocorrelated error terms. Journal of the American Statistical Association, 44(3):32-61.

DeGroot, M. H. (1970). Optimal Statistic Decision. McGrall-Hill, New York. 
Dillon, J. L. \& Scandizzo, P. L. (1978). Risk attitudes of subsistence farmers in northeast of Brazil: A sampling approach. American Journal of Agricultural Economics, 60:425-435.

Doreian, P. (1980). Linear models with spatially distributed data: Spatial disturbance or spatial effects? Sociological Methods and Research, 9:29-66.

Ellis, F. (1993). Peasant Economics: Farm Household and Agrarian Development. Cambridge University Press, New York.

Faminow, M. D., Dahl, C., Vosti, S., Witcover, J., Oliveira, S., \& Carpentier, C. (1999). Smallholder risk, cattle and deforestation in the western Brazilian Amazon. World Animal Review, 93:16-23.

Fearnside, P. M. (1985). Environmental change and deforestation in the Brazilian Amazon. In Change in the Amazon Basin: Man's Impact on the Forest and Rivers, pages 70-89. Manchester University Press, Manchester.

Fujita, M., Krugman, P., \& Venables, A. (1999). The Spatial Economy: Cities, Regions, and International Trade. The MIT Press, Cambridge.

Godoy, R., Frank, J. R., \& Cláudio, M. A. (1998). Adoption of modern agricultural technologies by lowland indigenous groups in Bolivia: The role of households, villages, ethnicity and markets. Human Ecology, 26(3):351-369.

Godoy, R., O’Neill, K., Groff, S., Kostishack, P., Cubas, A., Demmer, J., McSweeney, K., Overman, J., Wilkie, D., Brokaw, N., \& Martínez, M. (1997). Household determinants of deforestation by amerindians in Honduras. World Development, 25(6):977-987.

Homma, A. K. O., Walker, R., Carvalho, R. A., Conto, A. J., \& Ferreira, C. A. P. (1996). Razões de risco e rentabilidade na destruição de recursos florestais: O caso de castanhais em lotes de colonos no sul do Pará. Revista Econômica do Nordeste, 27(3):515-535.

Instituto Brasileiro de Geografia e Estatística (1996). Censo demográfico. Rio de Janeiro, IBGE.

Jones, D., Dale, V. H., Beauchamp, J. J., Pedlowski, M. A., \& O'Neill, R. V. (1995). Farming in Rondonia. Resource and Energy Economics, 17:155-188.

Kaimowitz, D. \& Angelsen, A. (1998). Economic Models of Tropical Deforestation: A Review. Center for International Forestry Research, Bogor, Indonesia. 
Liu, D. S., Iverson, L. R., \& Brown, S. (1993). Rates and patterns of deforestation in the Philippines: Applications of geographic information system analysis. Forest Ecology and Management, (57):1-16.

Mahar, D. J. (1989). Government Policies and Deforestation in Brazil's Amazon Region. World Bank, Washington.

Mamingi, N., Chomitz, K. M., Gray, D. A., \& Lambim, E. F. (1996). Spatial patterns of deforestation in Cameroon and Zaire. Washington, World Bank, 37p. (Research Project on Social and Environmental Consequences of GrowthOriented Policies. Policy Research Department, Working Paper, 8.).

McCracken, S. D., Siqueira, A., Moran, E. F., \& Brondizio, E. (1999). Domestic life course and land use patters in an agricultural frontier in Brazil. In Conference on Patterns and Processes of Land Use and Forest Change, pages 23-26. University of Florida.

Mertens, B. \& Lambim, E. F. (1997). Spatial modeling of deforestation in Southern Cameroon: Spatial disaggregation of diverse deforestation process. Applied Geography, 17:143-168.

Mertens, B. \& Lambim, E. F. (2000). Land-cover-change trajectories in southern Cameroon. Annals of the Association of American Geographers, 90(3):467-94.

Monela, G. C. (1995). Tropical rainforest deforestation, biodiversity benefits, and sustainable land use: Analysis of economic and ecological aspects related to the Nguru mountains. Dissertation (Ph.D.) - Agricultural University of Norway.

Mueller, B., Alston, L., Libecap, G., \& Schneider, R. (1994). Land, property rights and privatization in Brazil. The Quarterly review of Economics and Finance, 34:261-280.

Nakajima, C. (1969). Subsistence and commercial family farms: Some theoretical models of subjective equilibrium. In Wharton, C. R. E., editor, Subsistence Agriculture and Economic Development, pages 165-185. Aldine, Chicago.

Nelson, G. C. \& Hellerstein, D. (1997). Do roads cause deforestation? using satellite images in econometric analysis of land use. American Journal of Agriculture Economics, 79:80-88.

Ord, K. (1975). Estimation methods for models of spatial interaction. Journal of American Statistical Association, 70:120-126. 
Panayotou, T. \& Sungsuwan, S. (1994). An econometric analysis of the causes of tropical deforestation: The case of northeast Tailand. In Brown, K. \& Pearce, D., editor, The Causes of Tropical Deforestation, pages 192-210. University of British Columbia Press, Vancouver.

Pfaff, A. S. P. (1999). What drives deforestation in the Brazilian Amazon? Journal of Environmental Economics and Management, 37:26-43.

Pichón, F. J. (1993). Agricultural settlement, land use and deforestation in the Ecuadorian Amazon frontier: A microlevel analysis of colonist's land allocation behavior. Dissertation (Ph.D.) - University of North Carolina.

Pichón, F. J. (1997). Colonist land allocation decisions, land use and deforestation in the Ecuadorian Amazon frontier. Economic Development and Cultural Change, 35(3):707-744.

Reis, E. \& Guzman, R. (1994). An econometric model of the Amazon deforestation. In Brown, K. \& Pearce, D. e., editor, The Causes of Tropical Deforestation, pages 172-91. University of British Columbia Press, Vancouver.

Rudel, T. K. (1989). Is there a forest transition? deforestation, reforestation, and development. Rural Sociology, 63:533-552.

Serrão, E. A. S. \& Homma, A. K. O. (1993). Brazil. In Sustainable Agriculture and the Environment in the Humid Tropics, page 702. National Academy Press, Washington.

Serrão, E. A. S., Nepstad, D., \& Walker, R. T. (1996). Upland agricultural and forestry development in the Amazon: Sustainability, criticality and resilience. Ecological Economics, 18:3-13.

Singh, I., Squire, L., \& Strauss, J. (1986). The basic model: Theory, empirical results and policy considerations. In Singh, I. \& Squire, L. \& Strauss, J. e., editors, Agricultural Household Models. The Johns Hopkins University Press, Baltimore.

Thorner, D., Kerblay, B., \& Smith, R. E. F. (1986). The Theory of Peasant Economy. The University of Wisconsin Press, Madison.

Walker, R. T. (1999). The structure of uncultivated wilderness: Land use beyond the extensive margin. Journal of Regional Science, 39:387-410. 
Walker, R. T. \& Homma, A. K. O. (1996). Land use and land cover dynamics in the Brazilian Amazon: An overview. Ecological Economics, 18(1):67-80.

Walker, R. T., Moran, E., \& Anselin, L. (2000). Deforestation and cattle ranching in the Brazilian Amazon: External capital and household process. World Development, 8(4):683-699.

Walker, R. T., Perz, S., Caldas, M. M., \& Silva, L. G. T. (2002). Land use and land cover change in forest frontier: The role of household life cycle. International Journal of Regional Science, 25(2):169-199.

Walker, R. T. \& Solecki, W. D. (1999). Managing land use and land cover change: The New Jersey Pinelands biosphere reserve. Annals of the Association of American Geographers, 89(2):219-236.

Wear, D. N. \& Bolstad, P. (1998). Land use changes in southern Appalachian landscapes: Spatial analysis and forecast evaluation. Ecosystems, 1:575-594. 